\title{
The "Acceptance" in the Elaboration of Mourning in Oncological Diseases: Definition, Theoretical Models and Practical Applications: Needs Analysis and Subjective Oncological Reality
}

\author{
Giulio Perrotta* \\ Department of Criminal and Investigative Psychology, Italy \\ *Corresponding author: Giulio Perrotta, Department of Criminal and Investigative Psychology, Italy
}

\section{ARTICLE INFO}

Received: 幽 August 28, 2019

Published: 絊 September 03, 2019

Citation: Giulio P. The "Acceptance" in the Elaboration of Mourning in Oncological Diseases: Definition, Theoretical Models and Practical Applications: Needs Analysis and Subjective Oncological Reality. Biomed J Sci \& Tech Res 21(1)2019. BJSTR. MS.ID.003547.

\begin{abstract}
Starting from the general concept of mourning and oncological disease, the topic has been expanded by re-elaborating the main theoretical models and practical applications, to further explore aspects related to subjective needs and psychological techniques of cancer patient management, with particular care and attention to the ACT model with respect to the acceptance of the disease status.
\end{abstract}

Keywords: Mourning; Acceptance; Cancer; Act Model; Cancer Patient

\section{Introduction}

\section{Definition, Theoretical Models and Practical Applications}

Definition and Clinical Context: The "mourning" is the feeling of intense pain that one feels for the loss, in general, of a loved one, but it can also extend to all those hypotheses of the conclusion of an emotional and sentimental relationship with lasting and stable relationships or even to relationships that - beyond time present clear elements of relational dysfunction. Consequently, the "elaboration of mourning" consists in the work of assumption of emotional awareness of the meanings, of the experiences and of the social processes linked to the loss of the "relational object", that is of the person (relative or friend) with whom one was developed a significant emotional bond, interrupted by the death (or loss) of the same. The process of mourning, based on the intensity of the emotional bond, its modalities, and different protective or risk factors, can vary from six to twenty-four months, especially in the hypotheses of loss of a primary relational figure (example: parents, children, partners). It is, therefore, a conscious elaboration of a subjective nature [1]. In clinical psychology, instead, mourning whose elaboration is interrupted, profoundly slowed down or petrified due to the substantial impossibility of accepting the emotional meaning of relational loss is defined as "complicated mourning"; in this hypothesis, what is the discomfort or emotional pain (even acute) that usually accompanies every grief, can widen up to take on psychopathological forms; near the "complicated" form, we find the "traumatic" one, which is the substantial emotional suffering due to the loss: it is established starting from a critical event, such as an unexpected and sudden death (such as a car accident or a suicide) and even if it presents profiles of greater complexity than "normal" mourning, traumatic mourning does not necessarily result in complicated mourning; however, it is often the basis of a psychological trauma. In situations of complicated mourning and traumatic mourning, the intervention of a therapist who has specific training in psychotropic or psychotraumatology is fundamental [2].

Theoretical Models: On the basis of the aforementioned premises, over the last century, various models of mourning have been proposed, taking into consideration the acceptance as an ideal conclusion of the path of awareness of one's own emotions and feelings [1]: 
a) Freud considered mourning a psychogenetic paradigm of melancholia, or the loss of something external to Self [3];

b) Bowlby, distinguishing mourning in acute, chronic and prolonged, structured it in 4 precise phases:

c) Protest (lived with rejection, anguish and fear);

d) Nostalgia (lived with depression);

e) Despair (experienced with restlessness and anger);

f) Reorganization [4]

A. Parkes, distinguishing mourning in "physiological" (normal) and "pathological" (in its typologies: "chronic" - intense -, "delayed" - post-intense - and "inhibited" - lack of reaction -), the structured in 7 phases:

i. Denial/Avoidance;

ii. Alarm reactions (anxiety);

iii. Research of the irrational;

iv. Anger/Guilt;

v. Sense of inner emptiness;

vi. Adoption of traits/gestures typical of the deceased;

vii. Acceptance/Resolution [5].

B. Rando, who distinguished mourning in "physiological" and "pathological", where the second was nothing but the expression of the impairment, distortion or failure of one of the typical phases of physiological mourning, namely:

i. Recognize the loss;

ii. React to separation;

iii. Re-live the deceased and the relationship that was with him;

iv. Give up the past and what has been;

v. Reorganize one's life according to the loss;

vi. Reinvest feelings in other people [6].

Klüber-Ross, perhaps the most famous model of "mourning" of the Swiss psychiatrist and founder of psychopathology, which identifies the five phases (and not stages):

1) Negation Phase: "I cannot / want to believe it", "It's not really happening." In the first phase, the person tends to deny the loss. The rejection of reality can be considered a defence mechanism that has the function of saving the person suffering beyond measure;

2) Phase of Anger: "It's not fair", "What did I do wrong to deserve this?" During the second phase, the person experiences anger over the loss experienced as injustice. This is a very delicate passage in the psychological and relational process that can coincide with the moment of maximum request for help or with its exact opposite (total closure and withdrawal in oneself);

3) Negotiation Phase: "Overcoming this moment will make me stronger", "If I go out, I swear I won't make the same mistakes again". The third phase is one in which the person begins to take note of the irreversibility of the loss and to hypothesize, even in the alternation of moments of despair and hope, ways and strategies to regain control of one's life, evaluating what are the resources on which be able to count and new projects on which to invest their resilience skills;

4) Phase of Depression: "My life is hell", "There is no way out". The fourth phase brings with it an authentic awareness of the loss. The person focuses on everything he can no longer share with his former partner, unintentionally amplifying the level of suffering and generating a vicious circle that leads to a state of real depression. Typical symptoms of the moment are a headache, increase or loss of body weight, inability to concentrate, irritability, insomnia or excessive sleepiness, anger, frustration, persistent sadness and a desire to isolate oneself;

5) Acceptance Phase: "It's time to turn the page". The fifth and final phase coincides with the conclusion of the process of mourning, distinguishing itself from simple resignation. Although he may still incur emotional states of anger and depression (although in a decidedly lesser form than in the receding phase), the person is ready to make sense of what has happened, to inscribe it in the natural order of things, to accept the loss and to consider the possibility of an autonomous life project, looking to the future with renewed enthusiasm. This theoretical model has the following characteristics:

1. It was developed in 1969, from experience with people at the end of their lives and their families and operators.

2. The feelings experienced are typical responses to suffering.

3. The way to deal with pain is unique and subjective.

4. The phases are not stages. Therefore, there is no predetermined order, being able to alternate, recur or overlap.

5. There are five phases, but they do not necessarily have to be all through.

6. The way in which the news is communicated is of extraordinary importance, taking into account that every human being is composed of 4 quadrants: "physical" (love, affection and contact -typical of the first year of life); "Emotional" (emotions -typical in the 1-6 years-); "Intellectual" (knowledge -typical from 6 years onwards); "Spiritual / intuitive" (instinct, typical of adolescence).

7. The concept of death in children is structured in 3 phases: around 3 years, death is conceived as a temporary separation 
and attention is given to the body, with the loss that appears to be a malfunction of the body; after 5 years, death continues to be conceived as something external to the individual (e.g. a phantom man who takes away and makes disappear); around 9/10 years, the concept of death becomes more biological and realistic, practically permanent [1].

\section{Practical Applications}

In the practical sphere, it is necessary to distinguish three main hypotheses [2]:

a) The oncological patient during his therapeutic course, in an evolution phase with a favorable diagnosis;

b) The cancer patient during his therapeutic course, in an evolutionary phase with an unfavorable diagnosis;

c) The cancer patient during the final stage of the oncological disease, on a slope of poor diagnosis.

In the first hypothesis [2], the patient is in a time-lapse between the reception of the diagnosis and establishment of clinical treatment, in the presence of a probable favorable evolution, due to the specific elements of the case. In this hypothesis, the most suitable psychological treatment involves support to the patient to favor and increase (if necessary) his level of awareness of the disease and the role of the patient in the general picture. Also, in this hypothesis, it is not said that the patient's family needs support, even if an indepth interview is always recommended, in order to disseminate also the typical contents of primary and secondary prevention. In the second hypothesis [2], the patient finds himself in a time-lapse that always elapses between the receipt of the diagnosis and the establishment of clinical treatment, in the presence of a probable or certain unfavorable evolution, due to the specific elements of the case. In this hypothesis, the most suitable psychological treatment provides support to the patient to favor and increase (if necessary) his level of acceptance of the disease and the role of the patient in the general framework, in order to accompany him on his journey in the most serene and conscious as possible. Also, in this hypothesis, the need for a widening of the psychological support to the whole family of the patient is useful, in order to better facilitate the network of relations around the patient and disseminate the typical contents of primary, secondary and tertiary prevention.

In the third hypothesis [2], the patient is experiencing the last phases of his oncological pathology, with an undoubtedly unfavorable prognosis and is often approached to the "Palliative Care Centers" (Hospices) to help him concretely in the daily management of his personal and clinical needs. In this hypothesis, the most suitable psychological treatment involves supportive support for the patient, to accompany him to death in the most serene way possible. In this hypothesis, on the other hand, the need for a widening of the psychological support to the whole family of the patient, affected by the imminent loss of the loved one, is evident. And while on the one hand the patient and his needs are the main priority, on the other it is essential to pay attention to the management of human and professional dynamics related to the personnel working in the "Palliative Care Centers": in fact, $<<(.$.$) it is widely acknowledged that work in contact with suffering$ and death is a professional experience that involves operators on a personal, before a professional level [7-9]. Consequently, it is possible that more or less conscious identification processes are activated with the patient which, if not recognized and adequately managed, can favor the development of a form of emotional fatigue, described in literature as "compassion fatigue", or of a loss of sense with respect to one's work ("moral distress") or result in a more complicated condition of psycho-physical exhaustion, known as "burnout syndrome" [10-11].

The scientific literature has often questioned whether the confrontation with death the primary source of stress for palliative care workers and the studies is published in the last twenty years have produced conflicting results. An interesting review of the literature on the subject although dated, has shown that operators active in this area report less overall work stress when compared to other health workers who work with serious diseases, even if not necessarily terminal ones [12]. With the same work setting it was also shown that clinicians who feel insufficiently trained in communication and managerial skills have higher levels of stress than those who consider themselves more prepared in these specific areas; even insufficient confidence in patients 'psychological care has been more associated with physicians' burnout levels than the comparison with death has shown. An Australian study has suggested that the limited impact of the "death" factor on work stress is due to the fact that it is a problem that is mostly "taken on" by the Palliative Care organization model. It can be assumed that the specific organizational model of Palliative Care, as well as the training paths of the operators, perform an essential function of prevention or containment of stress-related work. More recent research has focused on the analysis of the relationship established between the Palliative Care operator and his / her work environment, identifying some specific factors that play a significant role in determining work-related stress.

These include: workloads (or the perception of not having enough time to perform one's duties well); control (or the feeling of not being able to exercise an adequate degree of control over the resources, personnel or organizational, necessary to carry out one's work); acknowledgments (the lack of adequate recognition of both economic and professional nature); relational well-being (the extent to which the individual feels a sense of personal connection with other work colleagues) and values (or the perception of a lack of coherence and discontinuity between one's work and one's reference values). The satisfaction or non-satisfaction of these requirements determine, together with the content of the work, the quality of the work experience. In light of the literature data briefly summarized, the interventions supporting the team will have to be planned after a careful analysis of the specific field from which the 
discomfort originates or develops, proposing consequently to the team, the most appropriate approach. (...)>> [13].

\section{In the Scientific Literature, The Techniques Used For "Staff Support" Interventions Are Listed:}

Staff Support Case: Based on the analytical support methodology, one of the main Staff Support models is born, the Staff Support Case, which uses the discussion of clinical cases like a working tool. The Case Support Staff supports the team is facing, in a dedicated space and time, the unavoidable criticalities of the care practice allowing that the experience of each professional does not remain confined to the individual dimension, but is instead "processable" in a group, transforming it into an opportunity for training growth for the entire staff. This is particularly important for severe or emblematic clinical cases, and for specific traumatic outcomes resulting from clinical events or care choices [7-14]. The case of an assisted person and the relative assistance pathway are analyzed within the group in order to evaluate the answers provided by the team to the complex needs of the patient and family members and the communicative-relational methods used. Crucial is the identification of any critical issues that have emerged and their causes (personal, professional and/or organizational). It follows the search for the most appropriate care approach as well as possible changes, both intra-individual and inter-individual, to be implemented within the team. It should be emphasized that the activity of the Palliative Care psychologist with the team operators in the Staff Support Case is not defined as an individual psychological assumption, but as an awareness-raising work, acquisition and promotion of knowledge, skills and attitudes of relational and/or communicative type and of "management of the Self" transversal to the different professions. The Staff Support Case, therefore, proposes itself on the one hand as a psychological revision intervention through the cases and on the other as continuous training of the care team.

Bereavement Debriefing: Healthcare professionals can more effectively address repeated exposure to situations related to dying if they find ways to understand and consolidate their experiences within broader meaning structures. Furthermore, the creation of a work environment that favor's the expression of spirituality also through the sharing of rituals or commemorations can increase the resilience of the operators [15]. The Bereavement debriefings are meetings aimed at supporting the staff with respect to particularly stressful or unexpected deaths, in which the various steps of the treatment process are reviewed in groups, leaving room for the expression of the experiences and emotions of each. Operators actively participating in these debriefings report a more exceptional ability to manage to grieve and maintain professional integrity [16]

Psycho-Educational Interventions: It is an organized set of interventions that combine informational moments with psychological support interventions aimed at improving knowledge and the management of specific problems. Psycho- educational interventions for the reduction of work stress are described by Bruneau BM and are aimed at preventing work stress through the teaching of cognitive coping skills and relaxation techniques while specific programs centered on end-of-life issues addressed in particular to the nursing staff, they are described by Boyle. These programs, conducted in groups, aim to improve the knowledge and professional skills of nurses who assist patients at the end of their lives. In recent years, literature has given ample space to psychoeducational interventions in the existential field that include the experiences that refer to the existential psychology of Viktor Frankl. The individual's ability to find a meaning capable of transforming intense stress situations into positive experiences can in fact act as a "profound motivational force" that improves the quality of life and well-being of individuals [17].

1) Existential Groups: They are based on guided reflections and Logotherapy exercises with the aim of improving the operators' ability to answer existential questions and support or promote spirituality as a resource to support the assistance process.

2) Psycho-Existential Training: they are centred on fears related to death and on the main themes of the psychology of dying. The goal is to help operators find answers and meanings in the face of the emotions generated by the theme of death.

3) Expressive/Narrative Forums: The expressive groups are aimed at providing emotional support and stimulating the growth processes of the Palliative Care operators. They are conducted through the use of expressive techniques, focused on feelings and emotions, and relaxation exercises [18] For example, the St Joseph's Hospice in London offers a monthly expressive forum for members of the entire staff, clinical and non-clinical, with the aim of offering a space to tell and freely express their emotional experiences related to daily care [19].

4) Art Therapy: Particular attention should be paid to some interventions that apply art therapy techniques and which have undergone considerable development in recent years. These include

a. Group art therapy programs: Include breathing exercises, guided visualizations, art, reflexive writing and discussions aimed at reducing psychological fatigue and increasing emotional awareness.

b. Art-making groups: The participants, working in pairs, create colored fabric panels. The objective is to stimulate and reflect on the value of collaboration in achieving common goals.

c. Music therapy groups: Group meditation practices with the use of music alongside the teaching of techniques for stress management are described by Hilliard R, while Wlodarczyk N proposes group exercises that through music they favor the processing of the mourning of the operators. 
5) Technical supervision: In the international scientific literature, on the other hand, the term "supervision" appears very rarely and, when it appears, mostly refers to an analytical rereading of clinical cases faced by the team often conducted by social workers.

The Subjective Approach to Cancer: The human and emotional experience of each person represents the cognitive substrate that allows the best or the worst adaptation to the traumatic event caused by the discovery of an oncological disorder; there are various factors involved, all of a psychological, biological and social nature, and the way in which the patient communicates the news also plays a fundamental role. Among the most common feelings, aroused by the news of the presence of a tumour, we have a sense of unreality, denial, confusion, disbelief, disorientation and anger. This is a very delicate and challenging phase for both the patient and his family [1]. The way to manage the "emotional crisis" generated by the medical diagnosis and the attitude towards the often-traumatic event will influence the type of psychosocial adaptation to the disease. The attitude and style of coping used will influence not only the quality of life after diagnosis but also compliance with medical treatments and the biological course of the disease [19]. In fact, there are more adaptive and less adaptive behaviors in dealing with one's health condition [20]. speaks of "abnormal disease behavior" emphasizing how perception, evaluation and consequently the behaviors implemented in relation to one's state of health may be inappropriate and maladaptive, although there was an adequate explanation on the part of the doctor nature of the disease, and an appropriate course of care has been defined, based on biological, psychological, social and cultural aspects. Abnormal disease behaviour includes both conditions characterized by an affirmation of the disease, such as hypochondria or conversion symptoms, and minimization or denial of symptoms. Coping represents a cognitive-behavioural modality with which an individual face a stressful event and its emotional consequences. The ability to cope with an existential crisis depends on several factors: on the type of pathology (symptoms and course), on the level of adaptation preceding illness, on the meaning of the existential threat, on cultural and religious factors, on the psychological set-up and from the personality, education and any psychiatric disorders present [21-24]. Among cancer patients, different coping strategies have been found in dealing with the stress linked to neoplastic disease[15].

\section{The Main Strategies Identified by Burgess (1988) are:}

a) Hopelessness/Helplessness, characterized by high levels of anxiety and depression, by the inability to implement cognitive strategies aimed at accepting the diagnosis, from the conviction of external control over the disease;

b) Combative Spirit, characterized by moderate levels of anxiety and depression, by numerous behavioural responses through which the patient tries to react positively and constructively to the situation, from the conviction of internal control over the disease;

c) Stoic Acceptance, with low levels of anxiety and depression, fatalistic attitude, from the conviction of external control of the illness;

d) Negation/Avoidance, in which both anxiety-depressive manifestations and cognitive strategies appear to be completely absent, in the patient's conviction of both internal and external control of the disease.

The perception of the control of an event [16], together with the desirability, are therefore fundamental factors in the cognitive evaluation of a stressor: the more the events are perceived as undesirable and uncontrollable the higher the probability of perceiving that event as stressful and the higher will be the probability of negative repercussions on health. Among the coping strategies most commonly used by cancer patients in addressing the emotional impact of the disease, denial/avoidance is found to be relevant in the diagnostic phase of the disease and is associated with low levels of emotional stress. Disease denial has been defined as a defence mechanism that allows distancing oneself from a threatening and worrying reality, "a conscious or unconscious refusal of a part or all the meaning of an event to ward off fear, anxiety or other unpleasant effects. A patient can deny the diagnosis, prognosis or severity of the disease, or he can ignore or forget what the doctor told him with the diagnosis or refuse to adhere to the proposed treatment. According to Breznitz, the negation that is activated in response to a threatening stimulus for one's own health determines a certain degree of distortion of reality and can relate to different aspects or parts of it. There are, therefore, seven types of negation that are articulated along a gradual continuum:

a) The denial of the personal meaning of the perceived threat;

b) The denial of urgency;

c) The denial of vulnerability or responsibility;

d) The denial of related emotions;

e) The negation of the affective meaning;

f) The denial of the presence of threatening information;

g) Denial of any kind of information.

A very interesting and debated aspect by scholars concerns the role or the function assumed by the negation on the course of medical and internist disorders as, depending on the stage of development of the disease in which the patient finds himself, the negation can have a positive or negative value, in relation to the contribution it can make to improving or worsening the medical condition. Studies in the literature show that the defensive mechanism plays an 
adaptive role in the initial stages of the disease because it protects the patient from fear, from the discomfort experienced when faced with a medical diagnosis. In studies conducted on women with breast cancer, there is a positive association between the denial of the effects of the disease (ie all the changes and the negative consequences of cancer) and lower levels of emotional distress as well as lower levels of anxiety and mood disorders.

The denial therefore through a "distortion" of reality, hiding from oneself the presence of cancer, helps to reduce the sense of oppression, of desperation, of fear, of helplessness that one experiences at the time of medical diagnosis, helping to preserve a positive self-image and self-esteem. Engel's (1977) biopsychosocial model underlines the importance of going beyond the strictly medical perspective and also considering the role of stressful life events, individual vulnerability to illness, disease behaviour, life experiences, the way of perceiving, assess and respond to your health status. It should also be emphasized that in some cases the defensive mechanism of denial can lead to the implementation of behaviors and attitudes that worsen the health condition: neglecting the symptoms and their meaning, not respecting adherence to medical therapies, delaying time the medical consultation makes the prognosis of the oncological disease more unfavorable. The timeliness of cancer diagnosis and compliance (adherence) to medical treatment are crucial in increasing the chances of a favorable resolution of the cancer, for this it is necessary to psychologically assist the patient from the moment of diagnosis, as is already being done in several Italian hospitals where there are psychologists who work alongside the doctor. Denial or denial could compromise the patient's adherence to medical prescriptions, drugs, laboratory tests, clinical controls and all this has a tremendous clinical relevance: fear, social pressures, a sense of responsibility but also the culture of belonging are all factors that could be connected with the denial of illness [17].

The psychological needs of the cancer patient in developing the concept of "acceptance" in a functional manner [18-21]. The disease destroys the balance of the mind-body unity, and the more the path and the experience of illness is tortuous and complicated, the more the dimensions of human reality, i.e. the physical, psychological and social dimensions, are affected. The research on the quality of life became relevant in medicine when it was realized that the clinical parameters usually used were not sufficient to evaluate the outcome of the treatments. In fact, in the evaluation of the results of medical treatments, it was understood that for the patient the variation of the symptomatic picture was only a small part, often the least important, in the personal satisfaction of his living conditions. To evaluate and measure the quality of life we rely on objective indicators, such as housing, income, social relations networks, education, social security, etc., and on subjective indicators, which are given from the perception, the evaluation and the satisfaction of the individuals of the various areas of their life (work, family, social relations, etc.). It can be said that objective indicators are part of the environmental and socio-economic aspects of life, while the subjective ones are cultural and psychological aspects. The subjective aspects of the quality of life, i.e. those of a psychological nature, are:

a. The perception of one's psychological well-being and of one's physical and social functioning (the appropriate use of the opportunities offered by society);

b. The individual subjective perception of one's existence;

c. The fulfillment of one's aspirations; d) self-evaluation of one's own realization in the context of one's own value system and in the various areas of life; e) global self-esteem. The neoplastic pathology is the most frequent cause of death and creates fear and anxiety in patients and relatives. The life expectancy can be long and with the resolution of a complete recovery, but it can also be short, and it is precisely here that the quality of life must be safeguarded as much as possible. The specificity of the tumour disease and the treatment undertaken for it are factors perceived and evaluated by the patient that affect his personality by defining specific moods and changes in his lifestyle.

d. The aims of the studies on the quality of life in the branch of oncology are the following:

I. To evaluate the therapy also with respect to psychosocial criteria;

II. Provide a better basis for making decisions on different treatments including quantitative and qualitative criteria for survival;

III. To help develop more adequate psychosocial care for patients with malignant disease".

e. In the field of oncology, Cella and Tulsky (1990) have identified ten subjective indicators of quality of life:

1. Physical concerns (symptoms and pain);

2. Functional skills (activity);

3. Family well-being;

4. Emotional well-being;

5. Spirituality;

6. Satisfaction with the treatment (including financial worries);

7. Future orientation (planning and hopes);

8. Sexuality/intimacy;

9. Social functioning;

10. Working functioning.

People, throughout their lives, are driven by the need to satisfy their biological and psychological needs. The prolonged dissatisfaction of these needs generates anxiety. If the individual does not listen to the anguish, the latter will go towards psychological 
suffering if not a mental illness. The most considerable anxieties are that of death, persecution, abandonment, loss of love, castration and guilt. The verification and management of the psychosocial and spiritual problems and needs of patients and their families, according to WHO said regarding the definition of palliative care, "is an integral part of the assistance plan and deserves the same attention paid to physical problems ". Attention to these needs and the interventions related to them also serve to help the patient rediscover the value and meaning of things, to find the meaning both of himself and of belonging to others, even when the disease with its progressive addiction and disability makes it difficult.

Cancer disease and its treatments have physical, psychological, social, emotional, spiritual and practical consequences in the lives of sick patients and their families. Information regarding these consequences and related needs is essential for three main reasons: to be able to plan an individualized care plan for the person, to make decisions on the allocation of resources and to identify potential areas to be developed to increase the quality of life perceived by the sick. The psychological needs of the cancer patient are influenced by the type of cancer, the stage of the disease and the type of treatment, so the implications and problems that arise are specific to each stage of the disease. However, they influence the patient's ability to cope with the pathology, his psychological stability and social support. report that although patients perceive the fact that they have a problem, they can also decide to endure this difficulty depending on the circumstances and not to report it as a need. Fitch also emphasizes that patients do not always want help from caregivers for multiple reasons, including patients' thinking that health professionals are too busy, the idea that the team does not care about specific types of problems, the belief that over time the problem vanishes or that there is nothing that can be done to deal with certain types of problems.

Consistently with the modern principles of the patient-centred care approach, needs are self-defined: they reflect the individual's desires rather than medical judgments or clinical interpretations of global well-being (which may show little consistency with the patient's priorities). In the context of the care relationship, in fact, the needs reflect the inconsistency between the individual perception of the support patient considered necessary and the real support provided. In the advanced stage of illness, the patient and the family realize that there is no longer any cure to avoid the inevitable end of life. In this phase of illness, the patient and his loved ones again encounter all the feelings, problems and difficulties already experienced, but amplified due to the impossibility of making use of the mitigation of hope. In approaching the terminal phase of the disease, the person and his family struggle to maintain a meaningful quality of life, face the decay of physical conditions, confront themselves with spiritual concerns and plan livelihood plans for the family members who will remain. Thanks to recent developments in medicine, the advanced stage of cancer is increasingly experienced as a chronic phase of the disease, with consequent changes in patients' needs. The psychophysical aggression of the disease breaks the mind-body balance of the individual, and the more the path of illness is complex and challenging, the more the dimensions of human existence (physical, psychological and social) are affected.

In fact, many patients have severe difficulties in adapting to the new conditions imposed by neoplastic disease, both physical and psychological and social. Realizing that you are no longer equal to before and therefore no longer able to recognize yourself as an individual, causes the patient to "fear being lost to themselves, anguish, despair". Following are feelings like abandonment, loneliness and marginalization, modulated by mental representations that the individual has on cancer [18]. The ominous meaning attributed to late-stage cancer can compromise the patient's state of safety, triggering emotional reactions that manifest themselves towards themselves and others. These emotional reactions produce a general state of disequilibrium, showing attitudes of internal instability or relational instability below. Internal instability due to the encounter with neoplastic disease, or the instability of one's own psyche and being, can be revealed through states of anxiety, feelings of guilt and shame, and can evolve up to self-punitive behaviors such as rejection of the treatments, the denial of the illness, or to underline resignation and passive behaviors. In fact, as [17]. reports, one of the psychological needs of the advanced cancer patient is precisely the need for safety [18]. indicate that one of the primary needs of the cancer patient in the advanced stage of illness is the need for safety, which is combined in the feelings of not feeling abandoned, of not feeling cheated, of receiving adequate assistance and that the care staff pay attention to on the specific difficulties of their situation Psychological problems can be anxious, depressive, organic, of anger and consequent to alterations of the body image "[18]. In this phase of illness, "anguishes of isolation, separation and death are exasperated"[18].

Symptoms such as depression and anxiety are present frequently and have a significant impact on the patient's quality of life. In fact, it has been shown that the lack of positive emotions is associated with the inability to engage in significant activities, the reduction of social and cognitive functioning. However, it must be emphasized that in view of the development of the stages of mourning, depression and anxiety can represent a fruitful moment in the process of adaptation and acceptance of loss, because they allow the individual to integrate the experience of illness into his personal life baggage [18]. divides the needs reported by patients into emotional needs and psychological needs. The former includes feeling depressed or depressed, feeling sad, feeling bored or useless and anxiety. Patients reported the problem of being uninformed about the possibility of psychological support, remaining at the mercy of feelings of fear, anguish, pain, anger and the need to confide. For which the need arises: to increase psychosocial support, increase the involvement of the support network, more information on the existing help offer, a telephone service to call in the most 
challenging moments. The most frequently detected emotions in advanced cancer patients in the they are fear and anxiety. Regarding the topic of fear, identifies five main fears related to the experience of cancer: death, disability, deterioration, dependence and loss of significant relationships.

Dealing with the fear of what will happen to yourself the fear of your own fate and the fear of the spread of cancer are needs (exposed in the articles as problematic but classified as needs) reported by this type of patient in different studies. In the list of twenty high or moderate specific needs reported most frequently by patients according to the need to address the fears related to the spread of cancer is in second place with $40 \%$ of respondents, and the need to face the fear of what will happen to yourself is in sixth place with $35 \%$ [18] set out the 15 needs most frequently stated by patients. The need to face fear about one's fate is in 13th place with $50.9 \%$, and the need to address the fears related to the spread of cancer is in 15 th place with $50.9 \%$ ever. In relation to the fear of facing one's fate, [6]. they report the anguish of the unknown, the fear of not succeeding and existential anxieties, such as the fear of nothingness, life and death. Fear of physical deterioration is also reported in these studies (33\%) [8] (57\%) [9] and the fear of physical disability that correlates with the fear of physical deterioration.

Another fear is that of pain. In the fear of pain and suffering characterizes $31 \%$ of advanced cancer patients Other significant fears are the fear of the sick about the fact that the results of the treatments are out of their control, the need to learn to have the situation under control (or the perception of this) and anxiety to undergo any treatment also highlight the difficulty of the patient in expressing his emotions subdivides the psychological needs of the cancer patient at an advanced stage of disease into seven primary needs, one of which is the need for self-esteem, particularly when physical disability increases; this means involvement in decisions regarding the patient's path of illness, but also the opportunity to give and receive [18] actively. This specific need is also mentioned by as the primary need of the cancer patient in the advanced stage of illness and expresses itself with the feeling of being appreciated, in the possibility of being able to maintain his own roles and in having decision-making power in his own path of illness [12].

Indeed, one of the problems reported by cancer patients and their families is the feeling of being poorly listened to and not sufficiently involved in their path of illness, and the requests presented to the health system in relation to this need are: higher listening and attention on the part of carers and be helped in the recognition and expression of their own needs and those of the family. Being able to express and express one's emotions has a crucial role in the path of adaptation and acceptance of illness and the relationship with reality and others. Moreover, it allows to release and channel towards the outside internal feelings of tension and discomfort that could not be managed independently without further worsening the discomfort [13]. The disequilibrium between mind and body of the patient caused by the psychophysical aggression of cancer also has repercussions on the temporal identity of the person, emphasizing the finiteness of life and attacking his emotional, working, family and social identity. The disease therefore also affects the socialization of the individual, an essential element of human life, looming over the patient's perception of being an integral part of the context in which he lives [18].

According to there is a fear of abandonment, of being a burden, of no longer having any value, of being revolting and of not having any more role than those preceding the disease Patients have the perception of residing in a passive role, confined to the role of a sick person, due to an insufficiently effective and sensitive communication, in their path of illness, for which they need: a sensitive and respectful communication of the peculiarity of the situation and of the subjects, to improve the methods of communication of the diagnoses, to verify that all the medical terms are understood and to be motivated and encouraged reports the psychological need for trust, expressed as the need for sincere and honest communication with family members and carers and the perception of receiving the best possible care [18]. In close connection with many of the needs referred to above, there is also the need for self-realization of the cancer patient at an advanced stage of illness, which is expressed through being able to express one's own projects (such as the future and the latest provisions) and the reassessment of one's existence [18-19]

\section{The Central Role of Active Listening}

To construct a plan of care shared with the patient, to identify realistic goals for it, and to be able to discuss its emotional reactions in front of the clinical picture, one must start primarily by creating a listening space, where the client can report his or her knowledge, its degree of awareness, its need for information, its expectations and its values. Only with a listening space, the patient has the possibility of being able to express and express their emotions. The manifestation and expression of emotions are crucial for the patient in order to adapt and accept the disease, but above all, to establish a relationship with reality and with others. In fact, if this externalization is lacking, there is a risk that the accumulation of internal feelings of tension and discomfort considerably worsens the patient's discomfort. The feelings that the patient might need to channel and communicate are:
a. Sadness;
b. Feeling useless;
c. Anxiety;
d. Fear of death;
e. Fear of disability;
f. Fear of deterioration;
g. Fear of addiction; 
h. Fear of the loss of significant relationships;

i. Fear about what will happen to himself;

j. $\quad$ Fear of being lost to oneself;

k. Fear of one's fate;

l. Fear of the spread of cancer;

m. Fear of pain;

n. Fear of suffering;

o. Fear of not making it, fear of existential nothingness;

p. $\quad$ Fear of being lost to oneself [17];

q. Distress towards the unknown;

r. Existential anxieties related to life and death;

s. Anguishes of separation, isolation and death;

The need to belong [17] is reported in the advanced stage of illness and can be expressed in having the possibility of being able to express one's thoughts and emotions, both the need for understanding, not only of symptoms and illness, but also in general of death and the possibility of obtaining a moment to discuss them [17]. Patients often experience difficulty in expressing their feelings and expressing and managing their own psychological needs (in fact many reports that they want help to identify and manage them). Furthermore, cancer patients report that they have often felt poorly listened to and not sufficiently involved in their disease pathway, and this fact makes us reflect a lot on the importance and the indispensability of increasing listening skills and techniques. Granting the patient space and time needed to express his specific needs allows him to ascertain his psychological state and is the elective support intervention that every operator should use in his communicative practice.

As mentioned above, it is of fundamental importance to evaluate the psychological state of the patient to ascertain that a state of physiological suffering does not become acute towards a state of pathological discomfort, and for this reason it is necessary to know the individual and relational representations and meanings of patients on cancer and the individual physiological adaptation mechanisms [17]. Recognizing and defining the feelings of patients and their representations about cancer allow us to evaluate the fears and the probability of the presence of psychological distress. The best tool to ascertain the psychological state of the patient is, therefore, the interview. Within this intervention, it is possible to discover the emotions that the patient experiences, but also the needs, problems and representations that characterize his experience. Moreover, the interview allows explaining not only what the needs of the patient are, but also which are the ones for which the latter wants help. In the context of a patient-centred approach to care, needs are self-defined and reflect the inconsistency that exists between the individual's perception of the supportive patient considered necessary and the real support provided by the healthcare team. Communication cannot exist if no one is willing to listen [18].

"Hearing is a physical act; listening is an intellectual and emotional action". Those who hear the sounds recognize the words, but the listener understands their meaning. To be considered an expert in interpersonal relationships, and therefore to be a specialist in the establishment of significant relationships, the specific skill required to possess is listening. It is essential in the advanced stage of illness to be close to the patient, to sit next to him and listen to his particular needs, even more so than to dialogue, so that he can express his fears, his worries, remember and relive his life and finally hope. This gives the patient the impression of being understood and allows him to trust us, thus entering into a personalized communication that considers his uniqueness, in which he can abandon himself and not be afraid of being left at the mercy of himself and his pathology. Feelings such as abandonment, loneliness and marginalization are often present in cancer patients in this phase of illness.

Listening to the patient's history is one of the goals the care provider must set himself to offer patient-centred care. In fact, the patient's history is what he has more than significant and characterizes his entire existential journey. Focusing on listening and understanding its importance allows us to improve communication, to have control over the context, to show our interest and to be a better professional. Van Ooijen and Charnock divide therapeutic listening into three main phases: "receiving and understanding, communicating what has been understood and getting from the other person the awareness of having been heard and understood". To have an effective communication it is essential to privilege active listening techniques and reflective listening at the expense of passive listening (hearing only words without giving any importance) and selective listening (filtering the message by hearing only what interests you), which they are inefficient and non-productive. The skills of active listening and reflective listening, attempting to create a bond and a meaningful and therapeutic relationship, include: the reflective meaning (that is, the repetition of the concepts or critical words expressed by the interlocutor through the formulation of questions or the use of a selective echo, useful for making the other person understand that the message has been understood, to encourage her to investigate particular elements of the speech or to provide specific answers to what she really wants to know), the reflective feeling (or focus on feelings underlying the content of the message, thus promoting an increase in awareness of hidden, unrecognized or unacknowledged emotions, silence, questions, paraphrases, summaries and the ability to listen to oneself. The use of these skills allows the patient to be able to express his emotions in a safe, welcoming, empathic, non-judgmental context and to be able to re-elaborate them and become aware of them thanks to listening to the reformulation done by the doctor. 
One of the primary psychological needs of the cancer patient at an advanced stage of illness is necessarily the need for acceptance "regardless of mood, social relationships and appearance". Accepting the patient also means welcoming his feelings without falling into judgment, thus giving the impression to the patient of the validity of his emotional and psychological expressions and highlighting our support. The patient must feel secure within the patient-caring relationship and must feel free to express himself without being judged. Only in this way will it be possible to create a satisfactory and useful therapeutic alliance for the purpose of continuity of care. Also, even silence can be active and creative when it is characterized by intimacy and harmony, which allow the patient to feel at ease and to be able to deepen his own self and that of the carer with greater awareness. Furthermore, silence can encourage the patient to continue talking without being interrupted, allowing him to tell his story with his rhythm and encourages the creation of a safe space where the person can express himself without being blocked or hindere.

However, silence can also be harmful, and therefore passive, destructive and uncomfortable, if the patient perceives tension, opposition and hostility. The patient may not feel comfortable in the silence and feel uncomfortable in it. In order to use active listening techniques, and thus increase one's listening skills, it is necessary to manifest participation during communication, to know both verbal and non-verbal communication, to be predisposed to listen, to know one's own emotional filters that influence the understanding of the message received, do not judge. Mental obstacles, unlike emotional filters that alter and select the message, completely block the reception of information. The most frequent are fear, worry, lack of preparation, fantasizing, boredom, lack of self-esteem and anger. However, note how the obstacles to listening largely coincide with the obstacles to the assessment of psychological and emotional needs by the carers. The lack of confidence in talking about emotions, the worry of not being able to manage the strong emotional reactions, the limited trust in psychological interventions and the conviction of not having enough skills to be able to intervene in a non-somatic area, are elements that unconsciously ensure that the carer does not implement a participatory and active listening. Finally, "finding oneself in others" can help the patient contain fear and find a new continuity of the ego. Only at this point can the awareness of death be the source of a new awareness of the value of life.

\section{The Emotional Relationship Between "Acceptance" and "Resignation"}

Terminologically, the two words under consideration may seem synonymous; however, their differences are more than substantial [20-24]. Rogers, the father of humanistic psychology, argued that change was possible only after reaching full acceptance of who we are. "Acceptance" intended as a preparation for change and not for immobility. On this assumption the typical differences between these two states of mind are evident: in "acceptance", the subject is at peace with himself and the surrounding environment, does not experience negative emotional states such as anger or frustration and is ready to rise to a different level determined by the change of inner status; in the "resignation", the subject is not at peace with himself or with the surrounding environment, he still experiences negative emotional states such as anger or frustration which he nevertheless tries to suffocate by suffering the consequences and is not ready to rise to a different level (in how much is still linked to one or more components of the event that destabilized it).

In psychotherapy with a cognitive-behavioural approach, a series of indications are used that refer to the model called "Acceptance and Commitment Therapy" (ACT), where "acceptance represents one of the six fundamental processes that favour psychological and existential well-being, as opposed to experiential avoidance which is related to psychological rigidity and malaise. It is a model that has not been defined only for the macro-area of mourning and oncological diseases but is applied in dozens of hypotheses. In essence, acceptance consists of coming into psychological contact with undesirable private experiences in a full, open, and helpless way. It means allowing our emotions, our feelings, our feelings to be as they are, regardless of whether they are pleasant or painful; it consists in opening oneself by creating a space for them, making sure that they are free to come and go in a natural way, dropping the "struggle" that is generally undertaken against what is experienced as unpleasant. A term that can be used instead of acceptance is availability (to be available).

In the avoidance of one's own painful internal experiences, on the other hand, one often has the sensation of feeling better but immediate relief is associated with the escape or suppression of an emotion, a situation or an aversive interaction is such a seductive prize that almost all Human beings often fall into experiential avoidance. It is no coincidence that Russ Harris, one of the leading exponents of the ACT, uses the word "accretion" instead of acceptance, precisely to emphasize its development potential. Thanks to acceptance, the energies used in a useless and counterproductive struggle against unpleasant emotions can be released, so that they can be used in actions that are useful and consistent with your values.

The six key processes underlie two macro-areas. The two macro-areas in question are:

1. The "processes of mindfulness and acceptance"; and

2. The "processes of behavioural modification and action committed according to values". Also, so:

A. The crucial first target process of the ACT intervention is experiential avoidance. Experiential avoidance is that set of strategies that we implement to control or alter our internal experiences (thoughts, emotions, feelings or memories), even when this causes behavioural damage. Attempts to control anxiety, thoughts to control other thoughts (e.g., brooding), to try in every way not to think or not to remember a pain through harmful and dysfunctional behaviours. Experiential avoidance also 
materializes in attempts to escape or control external experience, such as avoiding anxiety, avoiding conflicts or expressing anger. The functional equivalent of experiential avoidance in the ACT is called "Acceptance" and can be defined as "leaving space" or "opening up to the experience" of painful emotions and painful thoughts and memories. In this sense, the ACT therapy aims to promote some acceptance trends: a) not to judge our internal (and external) experiences with a malicious look by the inquisitor of ourselves; b) welcome the emotional states and give them the "informative" importance they deserve; c) weaken the power of thoughts about our behavior and our daily experience.

B. The second fundamental process for the ACT is cognitive fusion. In the ACT, the term "cognitive fusion" refers to the tendency of human beings to be captured, "harnessed" by the contents of their thoughts. The principle that justifies the dysfunctionality of this "connection to thoughts" is summarized in the following sentence: It is not so much what we think about creating problems and suffering but the way in which we relate to what we think. When we are "fused" with our thoughts, especially the dysfunctional ones, we forget that we are interacting with thought and not with a real event, a bit as if our thoughts and our assessments of reality lived in our place. The virtuous counterpart of cognitive fusion, in the ACT, is the Defusione. Therefore, it is of primary importance to intervene not on the contents of dysfunctional thoughts, but on how the individual relates to his own thoughts. In this way, we focus on the attitude towards our thoughts and not on the thoughts themselves. For example, doing dysfunctional thoughts of a depressive or anxious type does not make much difference from the ACT point of view: it is the influence they have on the life of the individual (dictated by the attitude that the individual himself has towards their own depressive/anxious thoughts) to define their impact on individual suffering.

C. The crucial third process on which the Acceptance Commitment Therapy focuses is the "dominance of the past and the future on the present moment". This process can be defined as a set of difficulties in directing and maintaining attention to the present moment and changing the focus of attention between the various dimensions of one's existence. All the energies of the individual are concentrated on a "theme" or a difficulty, and from that topic it fails to come out, thus limiting its influence in one's life. Prototypical examples of past or future dominance of the present moment are brooding and depressive ruminations. At the moment when the past is ruminating or rumbling, these processes require much energy and focus all our attention on the process itself. The ACT intervention proposal is to promote contact with the present moment, to be psychologically present and available towards what happens in the present moment. We humans, for reasons related to a sort of "mental economy", naturally tend to perform many daily activities without paying attention to what we do. As if our actions were often managed by an "autopilot" that allows us to perform multiple activities simultaneously. Although on many occasions, this automaticity is useful and functional, there are several occasions in which to act automatically and to lose contact with what we are doing is harmful and dysfunctional for our life. Getting in touch with the present moment also means consciously choosing to bring one's attention to what is entering into me and into the external physical world at that precise moment.

D. The crucial fourth process of Acceptance Commitment Therapy is the "Conceptualized Self". We could define the conceptualized self as a set of "fusions" of definitions of ourselves that the mind of each of us tells us. These definitions usually touch on nuclear and relevant aspects for the definition of oneself and of oneself in relation to others. When this process is very present and can be harmful, one is strongly identified with the contents of one's mind. There are various forms that the conceptualized self can take in our daily lives. Some of the most frequent may be the "labels" we give ourselves. Let's think, for example, of being "the sick", "the unfortunate", "the nerd". On other occasions, the conceptualized self assumes the content of rigid fixations on specific problems, a block that leads to failing to grasp the evolution of experience. On other occasions, the conceptualized self can be characterized by "mergers" with some rigid and abstract/evaluative aspects of oneself. The self-conceptualized is a mask so glued to the skin of our face that we forget we are wearing it, and it becomes our eyes, our ears and our mouth. The conceptualized self contains an elaborate description of ourselves, to which we have become attached and which soon becomes so crystallized that we mistake it for absolute reality. So, a problem like an anxiety problem (but really right for any kind of difficulty) turns into the conceptualized self "I am an anxious", and no matter how many experiences I do where I did not experience that loud and frightening anxiety, I continue to describe myself verbally with "I am an anxious". What the ACT suggests as a functional counterpart of the conceptualized self is the Self as Context. The self as a context is a new point of view, sometimes never experienced, in which we learn to observe our internal and external experience from a privileged point of view, namely that of a "participant, kind, compassionate and curious observer" of his own experience. What ACT promotes is the observation of experiences as they occur, through a careful and conscious look at self-reflection of one's experience as it occurs. This could lead us to discover that we ourselves can learn to observe our experience as it happens, to look at it in a curious way and thus widen the horizon of possibilities, choices and recognize in this way which mask we wear.

E. The fifth is the "lack of contact with one's values". By this expression, we mean the set of difficulties linked to the identification of what is essential for the individual and would make his life meaningful and productive. In some cases, one can observe the confusion and emptiness of personal goals and individual goals. In essence, people who have difficulties in the "Lack of contact with their values" process have difficulty answering the question: "What do I want from life?" or "What is important to me?" or "What are my 
values? ". The term values in the ACT means something different from personal goals, concrete aspirations and morals. We could define values as "long-term desired qualities of life" (long-term desired quality of life. Values are what motivates people to change, to face difficult times. The difficult choices of our life are often made precisely by letting ourselves be guided by our values. Values are often final goals that guide the action committed to living. We can approach our values through sets of objectives, concrete, feasible (workable, one of the critical words of the ACT) and practicable.

F. The sixth pillar is the process that is called "lack of activity and commitment to pursue personal value". With this expression we refer to the phenomenon whereby even when we can become aware of our harmful mechanisms, of our mergers, of the masks we wear and moments of mindlessness, there is still an important step to be taken, that is, to commit ourselves to act and to pursue your own values. The most damaging obstacles to this commitment can be summarized in two categories of behaviour: impulsiveness and persistent avoidance. Both these behaviours lead to a life characterized by restriction of activities and rigidity of the behavioural repertoire. Always doing the same things, always avoiding the same situations is equivalent to not doing. The ACT proposal lies in the concept of "committed action": the term is used to define the personal action guided by one's own values; instead it foresees that the individual "reckon" with his own difficulties and fragility. Accepting and making contact with one's own frailties and guiding one's actions starting from one's own personal values allows one to pursue a meaningful and productive life, not without suffering, but satisfying and choice. In particular, it is essential for the Acceptance and Commitment Therapy the concept of workability, of "feasibility". An action committed and guided by its own purposes must also be feasible, actionable. In other words, the committed action consists of continually choosing to engage in actions in the direction of one's personal values, despite the difficult emotions that may be encountered along the way.

\section{Conclusion}

It is clear that acceptance, in cancer patients, represents both the final goal of a path of development of its pathological condition and a goal to be reached in order to face better the clinical challenges that await it, also in relation to the surrounding environment and to family network around the patient himself. Cancer, more than any other disease, requires a continuous and repeated adaptation effort from the patient. Psychological adaptation is aimed at preserving one's mental and physical integrity, addressing reversible disorders and integrating irreversible ones. It consists of a series of cognitive, emotional and behavioural reactions. At each stage of the disease, the psychological reactions are the result of a complex integration between the memory of past experiences, the perception of the future threat and the resources available, and it often happens to witness the comorbidity of the cancerous state with more or fewer psychopathologies less structured and rooted in the subject.
The treatment of the person affected by cancer must, therefore, have as its primary objective that of improving the quality of life and limiting the risk that the possible consequences of psychosocial suffering or the development of psychopathological conditions affect its future existence. The evaluation of the psychological and social dimensions, therefore, represents a constitutive element of the treatment of the person who has cancer that falls within the responsibility of each therapeutic figure, the general practitioner, the oncologist, the nurse, the psychiatrist and the psychologist, of the overall care team. Numerous studies have been emphasizing this need for years, confirmed by the results of epidemiological research on the prevalence of psychological disorders, on the evaluation of the quality of life, on the strategies useful for information and communication with the patient and the family, on the various possible models of social support, on specific educational and supportive techniques on a psychological level, on psychotherapeutic, psychopharmacological and integrated interventions.

The adaptation to the disease and to the treatments depends, therefore, to a large extent, on the quality of the relational approach of the care team, which is the author above all through the control of the side effects of the therapies, the control of the pain, of the anxious symptoms and depressive and everything that interferes with the quality of existence. This is possible through individualized management of the patient, through information on the various aspects of the pathology as well as through the assessment of his needs, his possibilities of choice, his family and social situation. This entails a personal investment for the treating team and requires an evident role overloading for anyone who attends the oncological structures, be they hospital wards, clinics inside the hospital, hospice or the same domicile as the sick person in the assistance services integrated.

The neoplastic pathology can have profound repercussions on the psychological, affective, family, social and sexual spheres of the patient and his family members. The early detection of psychosocial dimensions (screening of emotional distress, detection of the patient's needs and quality of life) is the prerequisite for identifying people who need targeted interventions. The activation, in the various neoplasms and phases of illness, of psycho-oncological pathways of prevention, treatment and rehabilitation of emotional distress - be they supportive or more specifically psychotherapeutic (individual, group, couple, family) - is therefore fundamental for the patient and his family [24]. Active listening, therapeutic talk, the use of standardized psychometric tools, targeted pharmacological therapies and psychotherapeutic counselling, mindfulness, biofeedback and hypnosis techniques seem to be the winning weapons to accompany the patient (and his family) in the steep path which will see him active.

\section{References}

1. Perrotta G (2019) General psychology. Luxco. 
2. Freud S (1976) Mourning and melancholia (in Metapsychology). Current considerations on war and death, Caducity, in Works pp. 1915-1017.

3. Bowlby J (2001) Attachment and loss. Basic Books, Torino, pp. 477.

4. Parkes, Murray C, Laungani P, Young, W (2015) Death and bereavement across cultures ( $2^{\text {nd }}$ edn.). London \& NY: Routledge.

5. Onofri A, Rosa LC, Il lutto (2016) Cognitive evolutionary psychotherapy and EMDR.

6. (2018) Psychologists Commission SICP. Psychological interventions to support the Palliative Care team.

7. Abendroth M, Flannery J (2006) Predicting the Risk of Compassion fatigue: A study of hospice nurses. J Hospice and Palliative Nur 8: 346356.

8. Aycock N, Boyle D (2009) Interventions to manage compassion fatigue in oncology nursing. Clin J Oncol Nurs 13(2): 183-191.

9. Blust L (2009) Health professional burnout. Journal of Palliative Medicine 12(7): 639-642.

10. Pereira SM, Fonseca AM, Carvahlo AS (2011) Burnout in palliative care: A systematic review. Nurs Ethics 18: 317-326.

11. Keene EA, Hutton N, Hall B, Rushton C (2010) Bereavement debriefing sessions: An intervention to support health care professionals in managing their grief after the death of a patient. Pediatr Nurs 36(4): 185-189.

12. Lloyd-Williams M (2010) Psychosocial issues in palliative care. J Palliat Care 27: 287-295.

\section{ISSN: 2574-1241}

DOI: 10.26717/BJSTR.2019.21.003547

Giulio Perrotta. Biomed J Sci \& Tech Res

(C) $(9)$ This work is licensed under Creative

Submission Link: https://biomedres.us/submit-manuscript.php
13. Butow PN, Coates AS, Dunn SM (1999) Psychosocial predictors of survival in metastatic melanoma. J Clin Oncol 17: 2256-2263.

14. Butow PN, Coates AS, Dunn SM (2000) Psychosocial predictors of survival: Metastatic breast cancer. Ann Oncol 11: 469-474.

15. Soldini A (2017) Emotional and psychological needs of the cancer patient at an advanced stage of illness and nursing strategies to meet these needs. Bachelor Thesis. SUPSI-DEASS.

16. Bellani ML (2011) Psycho Oncology and palliative care. Nóos 17(2): 137 153.

17. Carpanelli I (2002) Italian association of nurses of oncology.

18. Dugger J (1999) Listening techniques: How to more effectively manage your communication and relationships at work, with friends, in the family Milano: Franco Angeli.

19. Hayes SC, Pierson H (2005) Acceptance and commitment therapy.

20. Harris R (2011) Fare ACT. Franco Angeli: Milano.

21. Hayes SC, Strosahl KD (2010) A practical guide to acceptance and commitment therapy. Springer: New York.

22. Broome A (1995) Health psychology. Process and applications, $\left(2^{\text {nd }}\right.$ edn.). Chapman \& Hall.

23. (2011) SIPO, Standards, options and recommendations for good psychooncology practice ( $2^{\text {nd }} \mathrm{edn}$.). Italian Journal of Psycho-Oncology 13: 2.

24. Perrotta G (2019) Clinical psychology. Luxco.

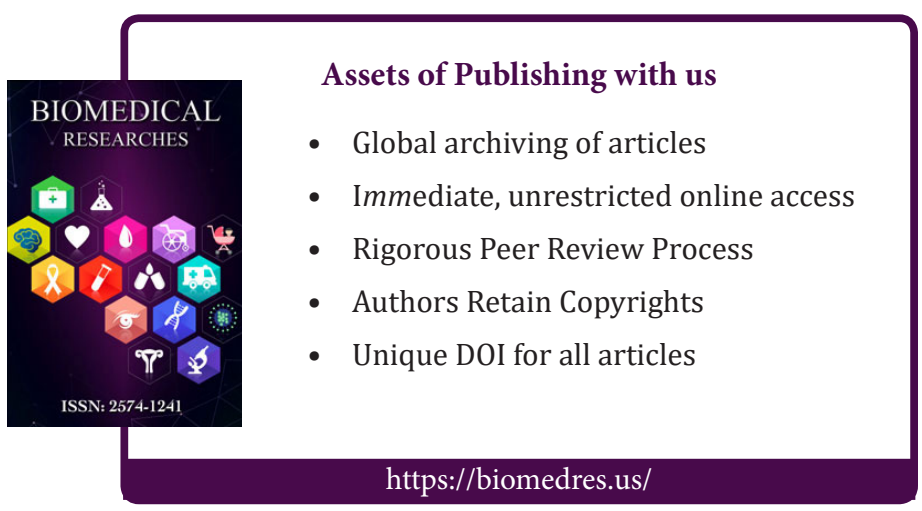

